\title{
Ulam stability of Caputo $q$-fractional delay difference equation: $q$-fractional Gronwall inequality approach
}

\author{
Rabia Ilyas Butt ${ }^{1}$, Thabet Abdeljawad ${ }^{2,3^{*}}$ (1), Manar A. Alqudah ${ }^{4}$ and Mujeeb ur Rehman ${ }^{1}$
}

\author{
"Correspondence: \\ tabdeljawad@psu.edu.sa \\ 2 Department of Mathematics and \\ General Sciences, Prince Sultan \\ University, Riyadh, Saudi Arabia \\ ${ }^{3}$ Department of Medical Research, \\ China Medical University, Taichung, \\ Taiwan \\ Full list of author information is \\ available at the end of the article
}

\begin{abstract}
In this article, we discuss the existence and uniqueness of solution of a delay Caputo $q$-fractional difference system. Based on the $q$-fractional Gronwall inequality, we analyze the Ulam-Hyers stability and the Ulam-Hyers-Rassias stability. An example is provided to support the theoretical results.
\end{abstract}

Keywords: Delay difference equation; $q$-fractional Gronwall inequality; Existence; Uniqueness; Ulam-Hyers stability; Ulam-Hyers-Rassias stability

\section{Introduction}

In the theory of differential equations, Gronwall's inequality is one of the most important tools. In 1919, for the first time, Gronwall worked on this type of inequality [1]. As time passed, many extensions of the Gronwall inequality have started to take part of the literature on mathematical inequalities. In 1935, Mikeladze published about the discrete fractional Gronwall inequality for the first time [2]. Gronwall's inequality is useful in the analysis of qualitative and quantitative properties of the ordinary and fractional dynamical systems. That is why it attracted many researchers to work on it. Haiping Ye et al. [3] presented a generalized Gronwall inequality and studied the dependence of the solution on the order and the initial condition of a fractional differential equation. Very recently, the authors in [4], proved a Gronwall inequality for the generalized proportional fractional operators. A class of stochastic Gronwall inequalities has been studied by Wang et al. in [5]. Luo et al. in [6] studied the uniqueness and novel finite-time stability of solutions of delay difference equations using the Gronwall inequality approach. Recently, Almeida et al. in [7] and Yassine et al. in [8] presented an extension of the fractional Gronwall inequality and used it in the qualitative analysis of the solutions to generalized fractional differential equations. For the category of fractional operators with nonsingular MittagLeffler kernels, a recent version and its application have been reported in [9].

Difference equations have appeared in mathematical modeling to describe many real life problems, e.g., queueing problems, electrical networks, economics, etc. For that reason, many researchers have proved discrete versions of Gronwall-type inequalities in fractional calculus and applied them to study the qualitative and quantitative properties of fractional difference equations [10-15]. Moreover, Gronwall's inequality is widely used for the analysis of stability of fractional differential as well as fractional difference equations. In one

(c) The Author(s) 2019. This article is distributed under the terms of the Creative Commons Attribution 4.0 International License (http://creativecommons.org/licenses/by/4.0/), which permits unrestricted use, distribution, and reproduction in any medium, provided you give appropriate credit to the original author(s) and the source, provide a link to the Creative Commons license, and indicate if changes were made. 
of the most recent works, Ameen et al. discussed the Ulam stability of delay fractional differential equations with a generalized Caputo derivative using a Gronwall inequality approach [16]. Kui Liu et al. [17] also presented Ulam-Hyers stability of solutions for differential equations with Caputo-Fabrizio fractional derivative with the help of Gronwall's inequality. For further assistance in stability analysis using the Gronwall inequality approach, one can follow the articles cited in [18-20]. As for the stability results without any Gronwall approach for the q-fractional systems, we refer to the first two works [21, 22]. Since stability, and specially Ulam-Hyers stability, is of high priority for researchers and has been studied for applied as well as mathematical problems, one can follow the most recent articles on stability cited in [23-25]. For recent operator and mathematical models, whose stability analysis is an open dilemma, we refer to [26-28].

In $q$-fractional calculus most probably the first article on a $q$-fractional Gronwall inequality was presented by Abdeljawad et al. in [29]. Later on, another new Gronwall inequality in $q$-fractional calculus was proved in [30], where the authors considered a nonlinear delay Caputo $q$-fractional difference system and discussed the uniqueness and estimates for the solutions of the system under consideration.

For $0<q<1$, time scale $\mathbb{T}_{q}$ is defined as $\mathbb{T}_{q}=\left\{q^{n}: n \in \mathbb{Z}\right\} \cup\{0\}$, where $\mathbb{Z}$ is the set of integers. If $n_{0} \in \mathbb{Z}$ and $a=q^{n_{0}}, \mathbb{T}_{a}$ can be written as $\mathbb{T}_{a}=[a, \infty)_{q}=\left\{q^{-i} a: i=0,1,2, \ldots\right\}$. Further define $\mathbb{I}_{\tau}=\left\{\tau a, q^{-1} \tau a, q^{-2} \tau a, \ldots, a\right\}$ and $\mathbb{T}_{\tau a}=[\tau a, \infty)_{q}=\left\{\tau a, q^{-1} \tau a, q^{-2} \tau a, \ldots\right\}$, where $\tau=q^{d} \in \mathbb{T}_{q}, d \in \mathbb{N}_{0}$, where $\mathbb{N}_{0}=\{0,1,2,3, \ldots\}$ and $\mathbb{I}_{\tau}=\{a\}$ with $d=0$ is the non-delay case. The objective of this paper is to study the existence, uniqueness and then analyze the Ulam-Hyers and Ulam-Hyers-Rassias stabilities of Caputo $q$-fractional difference equation with delay of the form

$$
\begin{cases}{ }_{q}^{c} \nabla_{a}^{\xi} u(t)=\mathcal{F}(t, u(t), u(\mathscr{G}(\tau t))), & t \in \mathbb{T}_{a}, \\ u(t)=\Phi(t), & t \in \mathbb{I}_{\tau},\end{cases}
$$

where $u: \mathbb{T}_{\tau a} \rightarrow \mathbb{R}, \mathcal{F}: \mathbb{T}_{a} \times \mathbb{R}^{2} \rightarrow \mathbb{R}, \mathscr{G}: \mathbb{T}_{a} \rightarrow \mathbb{T}_{a}, \Phi: \mathbb{I}_{\tau} \rightarrow \mathbb{R}$, and ${ }_{q}^{c} \nabla_{a}^{\xi}$ denotes the Caputo $q$-fractional difference operator of order $0<\xi<1$. To prove our main results, we use the $q$-fractional Gronwall inequality proved in [30].

This article is composed as follows: In Sect. 2, we present some basic definitions, notations, lemmas, and remarks that are important for proving our main results. In Sect. 3, we discuss the existence and uniqueness of solution of problem (1). Then, we discuss the Ulam-Hyers and Ulam-Hyers-Rassias stabilities of the above-mentioned problem. In the last section, examples are provided.

\section{Essential preliminaries and definitions}

In this section, we provide some basic concepts of $q$-fractional calculus that are essential to proving our main results. For more details on the theory of $q$-calculus and $q$-fractional calculus, we refer to [31-34] (and the references therein). For more remarkable basic articles in $q$-fractional calculus, we refer to [35-39]. The book [40] and [41] are also recommended for readers.

Definition 1 ([33]) For $u: \mathbb{T}_{q} \rightarrow \mathbb{R}$, the nabla $q$-derivative of $u$ is defined as

$$
\nabla_{q} u(t)=\frac{u(t)-u(q t)}{(1-q) t}, \quad t \in \mathbb{T}_{q}-\{0\} .
$$


Definition 2 ([33]) For $u: \mathbb{T}_{q} \rightarrow \mathbb{R}$, the nabla $q$-integral of $u$ is defined as

$$
\int_{0}^{t} u(s) \nabla_{q} s=(1-q) t \sum_{i=0}^{\infty} q^{i} u\left(t q^{i}\right)
$$

For $0 \leq a \in \mathbb{T}_{q}$,

$$
\int_{a}^{t} u(s) \nabla_{q} s=\int_{0}^{t} u(s) \nabla_{q} s-\int_{0}^{a} u(s) \nabla_{q} s .
$$

Definition 3 ([30, 33]) For $\xi \in \mathbb{R}$, we have:

(i) The nabla $q$-derivative of the $q$-factorial function with respect to $t$ is given by

$$
\nabla_{q}(t-s)_{q}^{\xi}=\frac{1-q^{\xi}}{1-q}(t-s)_{q}^{\xi-1}
$$

(ii) The nabla $q$-derivative of the $q$-factorial function with respect to $s$ is given by

$$
\nabla_{q}(t-s)_{q}^{\xi}=-\frac{1-q^{\xi}}{1-q}(t-q s)_{q}^{\xi-1}
$$

Definition $4([33,40])$ Let $u: \mathbb{T}_{q} \rightarrow \mathbb{R}$, then left $q$-fractional integral of order $\xi \neq$ $0,-1,-2,-3, \ldots$ and starting at $0<a \in \mathbb{T}_{q}$ is defined as

$$
{ }_{q} \nabla_{a}^{-\xi} u(t)=\frac{1}{\Gamma_{q}(\xi)} \int_{a}^{t}(t-q s)_{q}^{\xi-1} u(s) \nabla_{q} s
$$

where

$$
\Gamma_{q}(\xi+1)=\frac{1-q^{\xi}}{1-q} \Gamma_{q}(\xi), \quad \Gamma_{q}(1)=1, \quad \xi>0
$$

Definition $5([33,39])$ Let $u: \mathbb{T}_{q} \rightarrow \mathbb{R}$, where $0<\xi \notin \mathbb{N}$. Then the Caputo left $q$-fractional derivative of order $\xi$ is defined as

$$
{ }_{q}^{c} \nabla_{a}^{\xi} u(t)={ }_{q} \nabla_{a}^{-(n-\xi)} \nabla_{q}^{n} u(t)=\frac{1}{\Gamma_{q}(n-\xi)} \int_{a}^{t}(t-q s)_{q}^{n-\xi-1} \nabla_{q}^{n} u(s) \nabla_{q} s
$$

where $n=\lceil\xi\rceil+1$.

Lemma 1 ([33]) For $\xi>0$ and $u$ defined on suitable domain, we have

$$
{ }_{q} \nabla_{a}^{-\xi} \nabla_{a}^{\xi} \nabla_{a}^{\xi} u(t)=u(t)-\sum_{k=0}^{n-1} \frac{(t-a)_{q}^{k}}{\Gamma_{q}(k+1)} \nabla_{q}^{k} u(a)
$$

In particular, for $\xi \in(0,1]$, we have

$$
{ }_{q} \nabla_{a}^{-\xi c} \nabla_{a}^{\xi} u(t)=u(t)-u(a) .
$$


Lemma 2 ([33]) For $\xi \in \mathbb{R}^{+}$and $\mu \in(-1, \infty)$, we have

$$
{ }_{q} \nabla_{a}^{-\xi}(u-a)_{q}^{\mu}=\frac{\Gamma_{q}(\mu+1)}{\Gamma_{q}(\xi+\mu+1)}(u-a)_{q}^{\mu+\xi}, \quad 0<a<u<b .
$$

Definition 6 Eq. (1) is said to be Ulam-Hyers stable if there exists a real number $c$ such that, for all $\epsilon>0$ and for each $v(t)$ defined on $\mathbb{T}_{\tau a}$, satisfying the inequality

$$
\left|{ }_{q}^{c} \nabla_{a}^{\xi} v(t)-\mathcal{F}(t, v(t), v(\mathscr{G}(\tau t)))\right| \leq \epsilon, \quad t \in \mathbb{T}_{a},
$$

there exists a solution $u(t)$ defined on $\mathbb{T}_{\tau a}$ of Eq. (1) satisfying

$$
|v(t)-u(t)| \leq c \epsilon, \quad t \in \mathbb{T}_{\tau a} .
$$

Definition 7 Eq. (1) is said to be Ulam-Hyers-Rassias stable with respect to $\psi(t), \psi$ : $\mathbb{T}_{a} \rightarrow \mathbb{R}^{+}$if there exists a real number $c$ such that, for all $\epsilon>0$ and for each $v(t)$ defined on $\mathbb{T}_{\tau a}$ satisfying the inequality

$$
\left|{ }_{q}^{c} \nabla_{a}^{\xi} v(t)-\mathcal{F}(t, v(t), v(\mathscr{G}(\tau t)))\right| \leq \epsilon \psi(t), \quad t \in \mathbb{T}_{a},
$$

there exists a solution $u(t)$ defined on $\mathbb{T}_{\tau a}$ of Eq. (1) satisfying

$$
|v(t)-u(t)| \leq c \epsilon \psi(t), \quad t \in \mathbb{T}_{\tau a} .
$$

The following lemma is the key to proceeding.

Lemma 3 ([30]) Let $\xi>0, u(t)$ and $v(t)$ be nonnegative functions, $w(t)$ be a nonnegative and nondecreasing function for $t \in \mathbb{T}_{a}$ such that $w(t) \leq M$, where $M$ is a constant. If

$$
u(t) \leq v(t)+w(t)_{q} \nabla_{a}^{-\xi} u(t)
$$

then

$$
u(t) \leq v(t)+\sum_{k=1}^{\infty}\left(w(t) \Gamma_{q}(\xi)\right)_{q}^{k} \nabla_{a}^{-k \xi} v(t) .
$$

Corollary 1 ([30]) Under the hypothesis of Lemma 3, assume further that $v(t)$ is a nondecreasing function for $t \in \mathbb{T}_{a}$, then

$$
u(t) \leq v(t)_{q} E_{\xi}\left(w(t) \Gamma_{q}(\xi), t-a\right), \quad t \in \mathbb{T}_{a},
$$

where ${ }_{q} E_{\xi}(\lambda, t-a)=\sum_{k=0}^{\infty} \lambda^{k} \frac{(t-a)_{q}^{k \xi}}{\Gamma_{q}(k \xi+1)}$ is the q-Mittag-Leffler function.

\section{Main results}

In this section, we present our main results. First, we start by proving the existence and uniqueness of the solution of Eq. (1). Then, we proceed to analyzing the Ulam-Hyers and Ulam-Hyers-Rassias stabilities. 


\subsection{Existence and uniqueness results}

Consider the space $X=l_{\infty}\left(\mathbb{T}_{\tau a}\right)$ of bounded functions (sequences) on $\mathbb{T}_{\tau a}$, where $\mathbb{T}_{\tau a}=$ $[\tau a, \infty)_{q}$. The space $X$ is a Banach space with the norm defined by $\|z\|_{X}=\sup _{t \in \mathbb{T}_{\tau a}}|z(t)|$. In the following lemma we present the solution representation.

Lemma $4 u(t)$ satisfies Eq. (1) if and only if it satisfies the following q-sum equation:

$$
u(t)= \begin{cases}\Phi(t) & t \in \mathbb{I}_{\tau}, \\ \Phi(a)+{ }_{q} \nabla_{a}^{-\xi} \mathcal{F}(t, u(t), u(\mathscr{G}(\tau t))), & t \in \mathbb{T}_{a} .\end{cases}
$$

Proof For $t \in \mathbb{I}_{\tau}$, it is clear that $u(t)=\Phi(t)$ is the solution of Eq. (1). Now, for $t \in \mathbb{T}_{a}$, applying ${ }_{q}^{c} \nabla_{a}^{\xi}$ on both sides of Eq. (4), we get

$$
{ }_{q}^{c} \nabla_{a}^{\xi} u(t)={ }_{q}^{c} \nabla_{a}^{\xi} \Phi(a)+{ }_{q}^{c} \nabla_{a}^{\xi}\left({ }_{q} \nabla_{a}^{-\xi}\right) \mathcal{F}(t, u(t), u(\mathscr{G}(\tau t))) .
$$

Using the facts that ${ }_{q}^{c} \nabla_{a}^{\xi}($ constant $)=0$ and ${ }_{q}^{c} \nabla_{a}^{\xi}\left({ }_{q} \nabla_{a}^{-\xi}\right) u(t)=u(t)$, which can be seen by Theorem 7 in [33], we have

$$
{ }_{q}^{c} \nabla_{a}^{\xi} u(t)=\mathcal{F}(t, u(t), u(\mathscr{G}(\tau t)))
$$

On the other hand, from Eq. (4), for $t \in \mathbb{I}_{\tau}$, we have $u(t)=\Phi(t)$. Also, by applying $\nabla_{a}^{-\xi}$ on both sides of Eq. (1) and making use of Lemma 1, we get

$$
u(t)=u(a)+{ }_{q} \nabla_{a}^{-\xi} \mathcal{F}(t, u(t), u(\mathscr{G}(\tau t))),
$$

hence we get

$$
u(t)=\Phi(a)+{ }_{q} \nabla_{a}^{-\xi} \mathcal{F}(t, u(t), u(\mathscr{G}(\tau t))) .
$$

Now we present the following uniqueness theorem.

Theorem 1 Assume the following:

$\left(A_{1}\right) \mathcal{F}$ and $\Phi$ are continuous functions defined as $\mathcal{F}: \mathbb{T}_{a} \times \mathbb{R}^{2} \rightarrow \mathbb{R}$ and $\Phi: \mathbb{I}_{\tau} \rightarrow \mathbb{R}$;

$\left(A_{2}\right) \mathcal{F}$ satisfies the Lipschitz condition with $\mathscr{L}>0$ such that, for $t \in \mathbb{T}_{a}$,

$$
\left\|\mathcal{F}\left(t, u_{1}, u_{2}\right)-\mathcal{F}\left(t, v_{1}, v_{2}\right)\right\| \leq \mathscr{L}\left(\left\|u_{1}-v_{1}\right\|+\left\|u_{2}-v_{2}\right\|\right)
$$

$\left(A_{3}\right)$ The map $\mathscr{G}$ preserves the delay interval $\mathbb{I}_{\tau}$.

If $u(t)$ and $v(t)$ satisfy problem (1), then $u(t)=v(t)$.

Proof Let $z(t)=u(t)-v(t)$, then we have to show that $z(t)=0$. It is immediate that $z(t)=0$ for $t \in \mathbb{I}_{\tau}$. For $t \in \mathbb{T}_{a}$, we have

$$
z(t)={ }_{q} \nabla_{a}^{-\xi} \mathcal{F}(t, u(t), u(\mathscr{G}(\tau t)))-{ }_{q} \nabla_{a}^{-\xi} \mathcal{F}(t, v(t), v(\mathscr{G}(\tau t))) .
$$


If $t \in \mathbb{I}_{\tau^{-1}}=\left\{a, \ldots, \tau^{-1} a\right\}$, then (A3) forces $z(\mathscr{G}(\tau t))=0$. Then, together with the other assumptions, it will imply that

$$
\begin{aligned}
\|z(t)\| & =\left\|\nabla_{q} \nabla_{a}^{-\xi} \mathcal{F}(t, u(t), u(\mathscr{G}(\tau t)))-{ }_{q} \nabla_{a}^{-\xi} \mathcal{F}(t, v(t), v(\mathscr{G}(\tau t)))\right\| \\
& \leq{ }_{q} \nabla_{a}^{-\xi}\|\mathcal{F}(t, u(t), u(\mathscr{G}(\tau t)))-\mathcal{F}(t, v(t), v(\mathscr{G}(\tau t)))\| \\
& \leq{ }_{q} \nabla_{a}^{-\xi} \mathscr{L}(\|u(t)-v(t)\|+\|u(\mathscr{G}(\tau t))-v(\mathscr{G}(\tau t))\|) \\
& \leq{ }_{q} \nabla_{a}^{-\xi} \mathscr{L}(\|z(t)\|+\|z(\mathscr{G}(\tau t))\|) \\
& \leq \frac{\mathscr{L}}{\Gamma_{q}(\xi)} \int_{a}^{t}(t-q s)_{q}^{\xi-1}\|z(s)\| \nabla_{q} s .
\end{aligned}
$$

An application of Corollary 1 will imply

$$
\|z(t)\| \leq 0{ }_{q} E_{\xi}\left[\mathscr{L} \Gamma_{q}(\xi), t-a\right]
$$

and hence $z(t)=0$ for $t \in \mathbb{I}_{\tau^{-1}}$. Next, for $t \in\left[\tau^{-1} a, \infty\right)_{q}$, we have

$$
\begin{aligned}
\|z(t)\| & =\left\|_{q} \nabla_{a}^{-\xi} \mathcal{F}(t, u(t), u(\mathscr{G}(\tau t)))-{ }_{q} \nabla_{a}^{-\xi} \mathcal{F}(t, v(t), v(\mathscr{G}(\tau t)))\right\| \\
& \leq{ }_{q} \nabla_{a}^{-\xi}\|\mathcal{F}(t, u(t), u(\mathscr{G}(\tau t)))-\mathcal{F}(t, v(t), v(\mathscr{G}(\tau t)))\| \\
& \leq{ }_{q} \nabla_{a}^{-\xi} \mathscr{L}(\|u(t)-v(t)\|+\|u(\mathscr{G}(\tau t))-v(\mathscr{G}(\tau t))\|) \\
& \leq \frac{\mathscr{L}}{\Gamma_{q}(\xi)} \int_{a}^{t}(t-q s)_{q}^{\xi-1}(\|z(s)\|+\|z(\mathscr{G}(\tau s))\|) \nabla_{q} s .
\end{aligned}
$$

If we let $\hat{z}(t)=\sup _{\beta \in \mathbb{I}_{\tau}}\|z(\mathscr{G}(\beta t))\|$, then we have

$$
\begin{aligned}
\hat{z}(t) & \leq \frac{\mathscr{L}}{\Gamma_{q}(\xi)} \int_{a}^{t}(t-q s)_{q}^{\xi-1}(\hat{z}(s)+\hat{z}(s)) \nabla_{q} s \\
& \leq \frac{2 \mathscr{L}}{\Gamma_{q}(\xi)} \int_{a}^{t}(t-q s)_{q}^{\xi-1} \hat{z}(s) \nabla_{q} s .
\end{aligned}
$$

Finally, Corollary 1 implies that

$$
\|z(t)\| \leq \hat{z}(t) \leq 0 \cdot_{q} E_{\xi}\left[2 \mathscr{L} \Gamma_{q}(\xi), t-a\right] .
$$

Hence again we have $z(t)=0$, i.e., $u(t)=v(t)$ for $t \in \mathbb{T}_{\tau a}$.

Now we present the following existence and uniqueness theorem for problem (1).

Theorem 2 In addition to assumptions $\left(A_{1}\right),\left(A_{2}\right)$, and $\left(A_{3}\right)$, let us assume that

$\left(A_{4}\right) \frac{2 \mathscr{L}(T-a)_{q}^{\xi}}{\Gamma_{q}(\xi+1)}<1$ for some $T>a$.

Then problem (1) has a unique solution in $\mathbb{T}_{\tau a}$.

Proof On the Banach space $X=l_{\infty}\left(\mathbb{T}_{\tau a}\right)$ define an operator as follows:

$$
\mathscr{T} u(t)= \begin{cases}\Phi(t), & t \in \mathbb{I}_{\tau}, \\ \Phi(a)+{ }_{q} \nabla_{a}^{-\xi} \mathcal{F}(t, u(t), u(\mathscr{G}(\tau t)), & t \in \mathbb{T}_{a} .\end{cases}
$$


For $t \in \mathbb{I}_{\tau}$, we have $|\mathscr{T} v(t)-\mathscr{T} u(t)|=0, u, v \in X$. Now, for $t \in \mathbb{T}_{a}$, we have

$$
\begin{aligned}
|\mathscr{T} v(t)-\mathscr{T} u(t)| & =\mid{ }_{q} \nabla_{a}^{-\xi} \mathcal{F}\left(t, v(t), v(\mathscr{G}(\tau t))-{ }_{q} \nabla_{a}^{-\xi} \mathcal{F}(t, u(t), u(\mathscr{G}(\tau t)) \mid\right. \\
& \leq{ }_{q} \nabla_{a}^{-\xi} \mid \mathcal{F}(t, v(t), v(\mathscr{G}(\tau t))-\mathcal{F}(t, u(t), u(\mathscr{G}(\tau t)) \mid \\
& \leq \mathscr{L}\left(\max _{t \in \mathbb{T}_{\tau a}}|v(t)-u(t)|+\max _{t \in \mathbb{T}_{\tau a}}|v(\mathscr{G}(\tau t))-u(\mathscr{G}(\tau t))|\right)_{q} \nabla_{a}^{-\xi}(1) \\
& \leq \frac{2 \mathscr{L}(t-a)_{q}^{\xi}}{\Gamma_{q}(\xi+1)}\|v-u\|_{X} \\
& \leq \frac{2 \mathscr{L}(T-a)_{q}^{\xi}}{\Gamma_{q}(\xi+1)}\|v-u\|_{X}, \quad t<T .
\end{aligned}
$$

Since $\frac{2 \mathscr{L}(T-a)_{q}^{\xi}}{\Gamma_{q}(\xi+1)}<1$, then the operator $\mathscr{T}$ is a contraction and by the Banach fixed point theorem there exists a unique fixed point. Clearly this unique fixed point is the unique solution of problem (1).

\subsection{Ulam-Hyers stability}

In the rest of what follows, we prove the Ulam-Hyers stability of problem (1).

Lemma 5 If a function $v(t)$ defined on $\mathbb{T}_{\tau a}$ is a solution of inequality (2), then $v(t)$ satisfies the following inequality:

$$
\left|v(t)-v(a)-{ }_{q} \nabla_{a}^{-\xi} \mathcal{F}(t, v(t), v(\mathscr{G}(\tau t)))\right| \leq \frac{\epsilon(t-a)_{q}^{\xi}}{\Gamma_{q}(\xi+1)}
$$

Proof As we know, $v(t)$ satisfies inequality (2) if and only if there exists a function $h(t)$ such that $|h(t)| \leq \epsilon$ and

$$
{ }_{q}^{c} \nabla_{a}^{\xi} v(t)-\mathcal{F}(t, v(t), v(\mathscr{G}(\tau t)))=h(t), \quad t \in \mathbb{T}_{a} .
$$

Now, applying $\nabla_{a}^{-\xi}$ on both sides of Eq. (5), we get

$$
\left({ }_{q} \nabla_{a}^{-\xi}\right)\left({ }_{q}^{c} \nabla_{a}^{\xi}\right) v(t)-{ }_{q} \nabla_{a}^{-\xi} \mathcal{F}(t, v(t), v(\mathscr{G}(\tau t)))={ }_{q} \nabla_{a}^{-\xi} h(t),
$$

or

$$
v(t)-v(a)-{ }_{q} \nabla_{a}^{-\xi} \mathcal{F}(t, v(t), v(\mathscr{G}(\tau t)))={ }_{q} \nabla_{a}^{-\xi} h(t) .
$$

Hence it follows that

$$
\begin{aligned}
\left|\left({ }_{q} \nabla_{a}^{-\xi}\right)\left({ }_{q}^{c} \nabla_{a}^{\xi}\right) v(t)-{ }_{q} \nabla_{a}^{-\xi} \mathcal{F}(t, v(t), v(\mathscr{G}(\tau t)))\right| & \leq{ }_{q} \nabla_{a}^{-\xi}|h(t)| \\
& \leq \epsilon_{q} \nabla_{a}^{-\xi}(1) \\
& =\frac{\epsilon(t-a)_{q}^{\xi}}{\Gamma_{q}(\xi+1)} \\
& =\frac{\epsilon(T-a)_{q}^{\xi}}{\Gamma_{q}(\xi+1)}, \quad t<T .
\end{aligned}
$$


Theorem 3 Under assumptions $\left(A_{1}\right),\left(A_{2}\right),\left(A_{3}\right)$, and $\left(A_{4}\right)$, Eq. (1) is Ulam-Hyers stable.

Proof Assume that $v(t)$ is a solution of inequality (2) and $u(t)$ is the unique solution of Eq. (1) satisfying the condition $u(t)=v(t)$ for $t \in \mathbb{I}_{\tau}$. Then we have

$$
u(t)= \begin{cases}v(t), & t \in \mathbb{I}_{\tau}, \\ v(a)+{ }_{q} \nabla_{a}^{-\xi} \mathcal{F}(t, u(t), u(\mathscr{G}(\tau t)), & t \in \mathbb{T}_{a} .\end{cases}
$$

For $t \in \mathbb{I}_{\tau}$, we have $|v(t)-u(t)|=0$. Now, for $t \in \mathbb{I}_{\tau^{-1}}=\left\{a, \ldots, \tau^{-1} a\right\}$, we have

$$
\begin{aligned}
|v(t)-u(t)|= & \left|v(t)-v(a)-{ }_{q} \nabla_{a}^{-\xi} \mathcal{F}(t, u(t), u(\mathscr{G}(\tau t)))\right| \\
\leq & \left|v(t)-v(a)-{ }_{q} \nabla_{a}^{-\xi} \mathcal{F}(t, v(t), v(\mathscr{G}(\tau t)))\right| \\
& +\left|{ }_{q} \nabla_{a}^{-\xi} \mathcal{F}(t, v(t), v(\mathscr{G}(\tau t)))-{ }_{q} \nabla_{a}^{-\xi} \mathcal{F}(t, u(t), u(\mathscr{G}(\tau t)))\right| \\
\leq & \left|v(t)-v(a)-{ }_{q} \nabla_{a}^{-\xi} \mathcal{F}(t, v(t), v(\mathscr{G}(\tau t)))\right| \\
& +\left|{ }_{q} \nabla_{a}^{-\xi}(\mathcal{F}(t, v(t), v(\mathscr{G}(\tau t)))-\mathcal{F}(t, u(t), u(\mathscr{G}(\tau t))))\right| \\
\leq & \left|v(t)-v(a)-{ }_{q} \nabla_{a}^{-\xi} \mathcal{F}(t, v(t), v(\mathscr{G}(\tau t)))\right|+\mathscr{L}_{q} \nabla_{a}^{-\xi}(|v(t)-u(t)|),
\end{aligned}
$$

where we have used $v(\mathscr{G}(\tau t))-u(\mathscr{G}(\tau t))=0$ for $t \in \mathbb{I}_{\tau^{-1}}$. Now, using Lemma 5 , we conclude that

$$
|v(t)-u(t)| \leq \frac{\epsilon(t-a)_{q}^{\xi}}{\Gamma_{q}(\xi+1)}+\mathscr{L}_{q} \nabla_{a}^{-\xi}(|v(t)-u(t)|)
$$

Since $\frac{\epsilon(t-a)_{q}^{\xi}}{\Gamma_{q}(\xi+1)}$ is a nonnegative and nondecreasing function $\forall t \in \mathbb{I}_{\tau^{-1}}$, then using Gronwall's inequality in Corollary 1, we see that

$$
|v(t)-u(t)| \leq\left[\frac{(t-a)_{q}^{\xi}}{\Gamma_{q}(\xi+1)^{q}} E_{\xi}\left(\mathscr{L} \Gamma_{q}(\xi), t-a\right)\right] \epsilon, \quad \forall t \in \mathbb{I}_{\tau^{-1}} .
$$

Now, for $t \in\left[\tau^{-1} a, \infty\right)_{q}$, following the same steps as mentioned above, we get

$$
\begin{aligned}
|v(t)-u(t)| \leq & \left|v(t)-v(a)-{ }_{q} \nabla_{a}^{-\xi} \mathcal{F}(t, v(t), v(\mathscr{G}(\tau t)))\right| \\
& +\mathscr{L}_{q} \nabla_{a}^{-\xi}(|v(t)-u(t)|+|v(\mathscr{G}(\tau t))-u(\mathscr{G}(\tau t))|) .
\end{aligned}
$$

Let $\hat{z}(t)=\sup _{\beta \in \mathbb{I}_{\tau}}|v(\mathscr{G}(\beta t))-u(\mathscr{G}(\beta t))|$, so we get

$$
\begin{aligned}
\hat{z}(t) & \leq \frac{\epsilon(t-a)_{q}^{\xi}}{\Gamma_{q}(\xi+1)}+\mathscr{L}_{q} \nabla_{a}^{-\xi}(\hat{z}(t)+\hat{z}(t)) \\
& \leq \frac{\epsilon(t-a)_{q}^{\xi}}{\Gamma_{q}(\xi+1)}+2 \mathscr{L}_{q} \nabla_{a}^{-\xi} \hat{z}(t) .
\end{aligned}
$$

Similarly, the use of Gronwall's inequality in Corollary 1 will lead to

$$
\hat{z}(t) \leq\left[\frac{(t-a)_{q}^{\xi}}{\Gamma_{q}(\xi+1)}{ }_{q} E_{\xi}\left(2 \mathscr{L} \Gamma_{q}(\xi), t-a\right)\right] \epsilon, \quad \forall t \in\left[\tau^{-1} a, \infty\right)_{q} .
$$


So we have

$$
|v(t)-u(t)| \leq \hat{z}(t) \leq\left[\frac{(t-a)_{q}^{\xi}}{\Gamma_{q}(\xi+1)}{ }_{q} E_{\xi}\left(2 \mathscr{L} \Gamma_{q}(\xi), t-a\right)\right] \epsilon, \quad \forall t,
$$

or

$$
|v(t)-u(t)| \leq\left[\frac{(T-a)_{q}^{\xi}}{\Gamma_{q}(\xi+1)}{ }^{q} E_{\xi}\left(2 \mathscr{L} \Gamma_{q}(\xi), T-a\right)\right] \epsilon, \quad \forall t<T
$$

That is,

$$
|v(t)-u(t)| \leq c \epsilon
$$

Hence completing the proof.

\subsection{Ulam-Hyers-Rassias stability}

This subsection is devoted to the Ulam-Hyers-Rassias stability of problem (1).

Theorem 4 In addition to assumptions $\left(A_{1}\right),\left(A_{2}\right),\left(A_{3}\right)$, and $\left(A_{4}\right)$, assume that

$\left(A_{5}\right)$ there exist a continuous function $\psi: \mathbb{T}_{a} \rightarrow \mathbb{R}^{+}$and $\lambda_{\psi} \in \mathbb{R}^{+}$such that

$$
{ }_{q} \nabla_{a}^{-\xi} \psi(t) \leq \lambda_{\psi} \psi(t)
$$

Then Eq. (1) is Ulam-Hyers-Rassias stable with respect to $\psi$.

Proof Let $v(t)$ be a solution of inequality (3). So we have

$$
-\epsilon \psi(t) \leq{ }_{q}^{c} \nabla_{a}^{\xi} \nu(t)-\mathcal{F}(t, v(t), v(\mathscr{G}(\tau t))) \leq \epsilon \psi(t) .
$$

Applying ${ }_{q} \nabla_{a}^{-\xi}$, we get

$$
-\epsilon_{q} \nabla_{a}^{-\xi} \psi(t) \leq\left({ }_{q} \nabla_{a}^{-\xi}\right)\left({ }_{q}^{c} \nabla_{a}^{\xi}\right) v(t)-{ }_{q} \nabla_{a}^{-\xi} \mathcal{F}(t, v(t), v(\mathscr{G}(\tau t))) \leq \epsilon_{q} \nabla_{a}^{-\xi} \psi(t) .
$$

Now, using assumption $\left(A_{5}\right)$, we get

$$
-\epsilon \lambda_{\psi} \psi(t) \leq v(t)-v(a)-{ }_{q} \nabla_{a}^{-\xi} \mathcal{F}(t, v(t), v(\mathscr{G}(\tau t))) \leq \epsilon \lambda_{\psi} \psi(t) .
$$

This implies

$$
\left|v(t)-v(a)-{ }_{q} \nabla_{a}^{-\xi} \mathcal{F}(t, v(t), v(\mathscr{G}(\tau t)))\right| \leq \epsilon \lambda_{\psi} \psi(t) .
$$

Let us consider $u(t)$ such that

$$
\begin{aligned}
& { }_{q}^{c} \nabla_{a}^{\xi} u(t)=\mathcal{F}(t, u(t), u(\mathscr{G}(\tau t))), \quad t \in \mathbb{T}_{a}, \\
& u(t)=v(t), \quad t \in \mathbb{I}_{\tau} .
\end{aligned}
$$


For $t \in \mathbb{I}_{\tau}$, we have $|v(t)-u(t)|=0$. Now, for $t \in \mathbb{I}_{\tau^{-1}}$, we have

$$
\begin{aligned}
|v(t)-u(t)|= & \mid v(t)-v(a)-{ }_{q} \nabla_{a}^{-\xi} \mathcal{F}(t, u(t), u(\mathscr{G}(\tau t)) \mid \\
\leq & \mid v(t)-v(a)-{ }_{q} \nabla_{a}^{-\xi} \mathcal{F}(t, v(t), v(\mathscr{G}(\tau t)) \mid \\
& +\mid{ }_{q} \nabla_{a}^{-\xi}(\mathcal{F}(t, v(t), v(\mathscr{G}(\tau t))-\mathcal{F}(t, u(t), u(\mathscr{G}(\tau t))) \mid .
\end{aligned}
$$

Using inequality (6) and the observation that $v(\mathscr{G}(\tau t))-u(\mathscr{G}(\tau t))=0$ for $t \in \mathbb{I}_{\tau^{-1}}$, we have

$$
|v(t)-u(t)| \leq \epsilon \lambda_{\psi} \psi(t)+\mathscr{L}_{q} \nabla_{a}^{-\xi}(|v(t)-u(t)|) .
$$

Now, by using Gronwall's inequality in Corollary 1, we conclude that

$$
|v(t)-u(t)| \leq \epsilon \lambda_{\psi} \psi(t)_{q} E_{\xi}\left(\mathscr{L} \Gamma_{q}(\xi), t-a\right)
$$

For $t \in\left[\tau^{-1} a, \infty\right)_{q}$, using the same steps as mentioned above, we have

$$
\begin{aligned}
|v(t)-u(t)| \leq & \left|v(t)-v(a)-{ }_{q} \nabla_{a}^{-\xi} \mathcal{F}(t, v(t), v(\mathscr{G}(\tau t)))\right| \\
& +\mathscr{L}_{q} \nabla_{a}^{-\xi}(|v(t)-u(t)|+|v(\mathscr{G}(\tau t))-u(\mathscr{G}(\tau t))|) \\
\leq & \epsilon \lambda_{\psi} \psi(t)+\mathscr{L}_{q} \nabla_{a}^{-\xi}(|v(t)-u(t)|+|v(\mathscr{G}(\tau t))-u(\mathscr{G}(\tau t))|) .
\end{aligned}
$$

Again, letting $\hat{z}(t)=\sup _{\beta \in \mathbb{I}_{\tau}}|v(\mathscr{G}(\beta t))-u(\mathscr{G}(\beta t))|$, we see that

$$
\hat{z}(t) \leq \epsilon \lambda_{\psi} \psi(t)+2 \mathscr{L}_{q} \nabla_{a}^{-\xi} \hat{z}(t)
$$

Finally, the use of Gronwall's inequality as in Corollary 1 implies that

$$
\begin{aligned}
\hat{z}(t) & \leq \epsilon \lambda_{\psi} \psi(t)_{q} E_{\xi}\left(2 \mathscr{L} \Gamma_{q}(\xi), t-a\right) \\
& \leq \epsilon \psi(t)\left[\lambda_{\psi q} E_{\xi}\left(2 \mathscr{L} \Gamma_{q}(\xi), t-a\right)\right] .
\end{aligned}
$$

So we have

$$
|v(t)-u(t)| \leq \hat{z}(t) \leq \epsilon \psi(t)\left[\lambda_{\psi q} E_{\xi}\left(2 \mathscr{L} \Gamma_{q}(\xi), t-a\right)\right], \quad \forall t
$$

or we may write it as follows:

$$
|v(t)-u(t)| \leq \epsilon \psi(t)\left[\lambda_{\psi q} E_{\xi}\left(2 \mathscr{L} \Gamma_{q}(\xi), T-a\right)\right], \quad \forall t<T
$$

This implies that

$$
|v(t)-u(t)| \leq c \epsilon \psi(t) .
$$

Hence Eq. (1) is Ulam-Hyers-Rassias stable. 


\section{Example}

The following example will illustrate Theorem 3.

Example 1 Let us consider the following problem:

$$
\begin{aligned}
& { }_{q}^{c} \nabla_{a}^{\frac{1}{2}} u(t)=\frac{\sin (u(t))+\sin (u(\tau t))}{200}, \quad t \in \mathbb{T}_{a}, \\
& u(t)=\cos 2 t, \quad t \in \mathbb{I}_{\tau} .
\end{aligned}
$$

$\left(A_{1}\right) \mathcal{F}\left(t, u, u^{*}\right)=\frac{\sin u+\sin u^{*}}{200}$ and $\Phi(t)=\cos 2 t$ are continuous functions.

$\left(A_{2}\right) \mathcal{F}$ satisfies the Lipschitz condition with Lipschitz constant $\mathscr{L}=\frac{1}{200}$ as follows:

$$
\begin{aligned}
\left|\mathcal{F}\left(t, u_{1}, u_{2}\right)-\mathcal{F}\left(t, v_{1}, v_{2}\right)\right| & \leq \frac{1}{200}\left(\left|\sin u_{1}-\sin v_{1}\right|+\left|\sin u_{2}-\sin v_{2}\right|\right) \\
& \leq \frac{1}{200}\left(\left|u_{1}-v_{1}\right|+\left|u_{2}-v_{2}\right|\right) .
\end{aligned}
$$

$\left(A_{3}\right) \mathscr{G}(\tau t)=\tau t$ preserves the delay interval $\mathbb{I}_{\tau}$,

$\left(A_{4}\right) \frac{2 \mathcal{L}(T-a)_{q}^{\xi}}{\Gamma_{q}(\xi+1)}=\frac{2\left(\frac{1}{200}\right)(T-a)_{q}^{\frac{1}{2}}}{\Gamma_{q}\left(\frac{1}{2}+1\right)}<1$ for some $T>a$.

Hence all the conditions are satisfied, so the problem under consideration is UlamHyers stable.

\section{Conclusion}

We summarize our concluding statements by the following:

1. Studying and analyzing fractional dynamical systems on different time scales are essential, and they have various applications in engineering and science. We have done our investigations on the quantum time scale.

2. We have proved the existence and uniqueness of solution for a delay Caputo $q$-fractional difference system depending on the Banach fixed point theorem and a recently proven version of q-Gronwall's inequality.

3. We have analyzed the Ulam-Hyers stability and the Ulam-Hyers-Rassias stability for q-fractional system under investigation.

4. An example is provided to support the Ulam-Hyers-Rassias stability proven theoretical result.

We have investigated our work in the frame of the classical Caputo q-fractional operators. We plan to continue this type of study for other types of fractional operators on the quantum time scale.

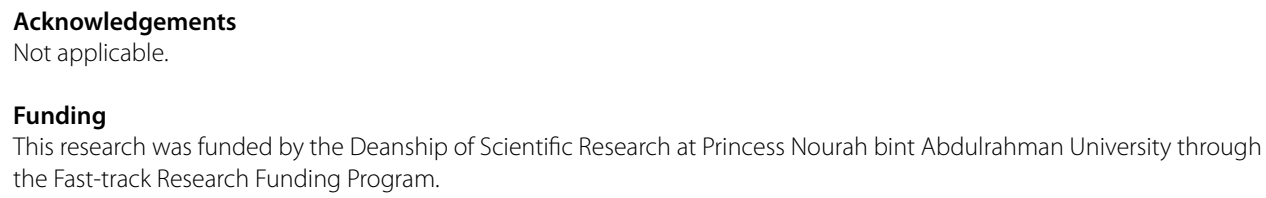

Availability of data and materials

Not applicable. 
Authors' contributions

All authors have made equal contribution to this article. All authors read and approved the final manuscript.

\section{Author details}

${ }^{1}$ Department of Mathematics, School of Natural Sciences, National University of Sciences and Technology, Islamabad, Pakistan. ${ }^{2}$ Department of Mathematics and General Sciences, Prince Sultan University, Riyadh, Saudi Arabia. ${ }^{3}$ Department of Medical Research, China Medical University, Taichung, Taiwan. ${ }^{4}$ Department of Mathematical Sciences, Princess Nourah bint Abdulrahman University, Riyadh, Saudi Arabia.

\section{Publisher's Note}

Springer Nature remains neutral with regard to jurisdictional claims in published maps and institutional affiliations.

Received: 6 October 2019 Accepted: 21 November 2019 Published online: 29 November 2019

\section{References}

1. Gronwall, T.H.: Note on the derivatives with respect to a parameter of the solutions of a system of differential equations. Ann. Math. (2) 20, 292-296 (1919) http://www.jstor.org/stable/10.2307/1967124

2. Mikeladze, Sh.E.: De la résolution numérique des équations intégrales. Bull. Acad. Sci. URSS VII, 255-257 (1935) (in Russian)

3. Ye, H., Gao, J., Ding, Y.: A generalized Gronwall inequality and its application to fractional differential equation. J. Math. Anal. Appl. 328, 1075-1081 (2007). https://doi.org/10.1016/j.jmaa.2006.05.061

4. Alzabut, J., Abdeljawad, T., Jarad, F., Sudsutad, W.: A Gronwall inequality via the generalized proportional fractional derivative with applications. J. Inequal. Appl. 2019, 101, 1-12 (2019). https://doi.org/10.1186/s13660-019-2052-4

5. Wang, X., Fan, S.: A class of stochastic Gronwall's inequality and its application. J. Inequal. Appl. 2018, 1 (2018). https://doi.org/10.1186/s13660-018-1932-3

6. Luo, D., Luo, Z.: Uniqueness and novel finite-time stability of solutions for a class of nonlinear fractional delay difference systems. Discrete Dyn. Nat. Soc. 2018, Article ID 8476285, 1-7 (2018). https://doi.org/10.1155/2018/8476285

7. Almeida, R., Malinowska, A.B., Odzijewicz, T.: An extension of the fractional Gronwall inequality. In: Advances in Non-Integer Order Calculus and Its Applications, 2019. https://doi.org/10.1007/978-3-030-17344-9_2

8. Adjabi, Y., Jarad, F., Abdeljawad, T.: On generalized fractional operators and a Gronwall type inequality with applications. Filomat 31(17), 5457-5473 (2017). https://doi.org/10.2298/FIL1717457A

9. Jarad, F., Abdeljawad, T., Hammouch, Z:: On a class of ordinary differential equations in the frame of Atangana-Baleanu fractional derivative. Chaos Solitons Fractals 117, 16-20 (2018)

10. Atici, F.M., Eloe, P.W.: Gronwall's inequality on discrete fractional calculus. Comput. Math. Appl. 64, 3193-3200 (2012). https://doi.org/10.1016/j.camwa.2011.11.029

11. Ferreira, R.A.C.: A discrete fractional Gronwall inequality. Proc. Am. Math. Soc. 140, 1605-1612 (2012). https://doi.org/10.1090/S0002-9939-2012-11533-3

12. Xu, R., Zhang, Y.: Generalized Gronwall fractional summation inequalities and their applications. J. Inequal. Appl., 2015, 242 1-10 (2015). https://doi.org/10.1186/s13660-015-0763-8

13. Fečkan, M., Pospisil, M.: Note on fractional difference Gronwall inequalities. Electron. J. Qual. Theory Differ. Equ. 2014, $64,1-18(2014)$

14. Abdeljawad, T., Al-Mdallal, Q.M.: Discrete Mittag-Leffler kernel type fractional difference initial value problems and Gronwall's inequality. J. Comput. Appl. Math. 339, 218-230 (2018). https://doi.org/10.1016/j.cam.2017.10.021

15. Alzabut, J., Abdeljawad, T.: A generalized discrete fractional Gronwall inequality and its application on the uniqueness of solution and its application on the uniqueness of solutions for nonlinear delay fractional difference system. Appl. Anal. Discrete Math. 12, 036 (2018) www.jstor.org/stable/90020603

16. Ameen, R., Jarad, F., Abdeljawad, T.: Ulam stability for delay fractional differential equations with a generalized Caputo derivative. Filomat 32(15), 5265-5274 (2018). https://doi.org/10.2298/FIL1815265A

17. Liu, K., Fečkan, M., ÓRegan, D., Wang, J.: Hyers-Ulam stability and existence of solutions for differential equations with Caputo-Fabrizio fractional derivative. Open Math., 7(4), 333, 1-14 (2019). https://doi.org/10.3390/math7040333

18. Wang, J., Fečkan, M., Zhou, Z.: Ulam's type stability of impulsive ordinary differential equations. J. Math. Anal. Appl. 395, 258-264 (2012). https://doi.org/10.1016/j.jmaa.2012.05.040

19. Wang, J., Zhou, Y., Fečan, M.: Nonlinear impulsive problems for fractional differential equations and Ulam stability. Comput. Math. Appl. 64, 3389-3405 (2012). https://doi.org/10.1016/j.camwa.2012.02.021

20. Wu, G.C., Baleanu, D., Zeng, S.D.: Finite-time stability of discrete fractional delay systems: Gronwall inequality and stability criterion. Commun. Nonlinear Sci. Numer. Simul. 57, 299-308 (2018). https://doi.org/10.1016/j.cnsns.2017.09.001

21. Jarad, F., Abdeljawad, T., Baleanu, D.: Stability of $q$-fractional non-autonomous systems. Nonlinear Anal., Real World Appl. 14(1), 780-784 (2013). https://doi.org/10.1016/j.nnorwa.2012.08.001

22. Jarad, F., Abdeljawad, T., Gundodu, E., Baleanu, D.: On the Mittag-Leffler stability of $q$-fractional nonlinear dynamical systems. Proc. Rom. Acad. 12(4), 309-314 (2011)

23. Khan, A., Khan, H., Gómez-Aguilar, J.F., Abdeljawad, T.: Existence and Hyers-Ulam stability for a nonlinear singular fractional differential equations with Mittag-Leffler kernel. Chaos Solitons Fractals 127, 422-427 (2019). https://doi.org/10.1016/j.chaos.2019.07.026

24. Khan, H., Abdeljawad, T., Aslam, M., Khan, R.A., Khan, A.: Existence of positive solution and Hyers-Ulam stability for a nonlinear singular-delay-fractional differential equation. Adv. Differ. Equ. 2019, 104 (2019). https://doi.org/10.1186/s13662-019-2054-z

25. Khan, A., Gómez-Aguilar, J.F., Khan, T.S., Khan, H.: Stability analysis and numerical solutions of fractional order HIV/AIDS model. Chaos Solitons Fractals 122, 119-128 (2019). https://doi.org/10.1016/j.chaos.2019.03.022 
26. Khan, H., Jarad, F., Abdeljawad, T., Khan, A.: A singular ABC-fractional differential equation with p-Laplacian operator Chaos Solitons Fractals 129, 56-61 (2019)

27. Khan, H., Li, Y., Khan, A., Khan, A.: Existence of solution for a fractional order Lotka-Volterra reaction-diffusion model with Mittag-Leffler kernel. Math. Methods Appl. Sci. 42(9), 3377-3387 (2019). https://doi.org/10.1002/mma.5590

28. Khan, A., Khan, T.S., Syam, M.I., Khan, H.: Analytical solutions of time-fractional wave equation by double Laplace transform method. Eur. Phys. J. Plus 134(4), 163 (2019). https://doi.org/10.1140/epjp/i2019-12499-y

29. Abdeljawad, T., Alzabut, J.: The $q$-fractional analogue for Gronwall-type inequality. J. Funct. Spaces Appl. 2013, Article ID 543839, 1-7 (2013). https://doi.org/10.1155/2013/543839

30. Abdeljawad, T., Alzabut, J., Baleanu, D.: A generalized $q$-fractional Gronwall inequality and its applications to nonlinear delay q-fractional difference systems. J. Inequal. Appl. 2016, 1 (2016). https://doi.org/10.1186/s13660-016-1181-2

31. Bohner, M., Peterson, A.: Dynamic Equations on Time Scales. Birkhäuser, Boston (2001)

32. Atici, F.M., Eloe, P.W.: Fractional q-calculus on a time scale. J. Nonlinear Math. Phys. 14(3), 333-344 (2007). https://doi.org/10.2991/jnmp.2007.14.3.4

33. Abdeljawad, T., Baleanu, D.: Caputo $q$-fractional initial value problems and a $q$-analogue Mittag-Leffler function. Commun. Nonlinear Sci. Numer. Simul. 16(12), 4682-4688 (2011)

34. Abdeljawad, T., Alzabut, J.: On Riemann-Liouville fractional $q$-difference equations and their application to retarded logistic type model. Math. Methods Appl. Sci. 41, 8953-8962, 1-10 (2018). https://doi.org/10.1002/mma.4743

35. Al-Salam, W.A.: q-Analogues of Cauchy's formula. Proc. Am. Math. Soc. 17, 182-184 (1952-1953)

36. Al-Salam, W.A., Verma, A.: A fractional Leibniz q-formula. Pac. J. Math. 60, 1-9 (1975)

37. Al-Salam, W.A.: Some fractional q-integrals and q-derivatives. Proc. Edinb. Math. Soc. 15, 135-140 (1969)

38. Agrawal, R.P.: Certain fractional $q$-integrals and q-derivatives. Proc. Camb. Philol. Soc. 66, 365-370 (1969). https://doi.org/10.1017/S0305004100045060

39. Rajkovic, P.M., Marinkovi, S.D., Stankovi, M.S.: Fractional integrals and derivatives in q-calculus. Appl. Anal. Discrete Math. 1(1), 311-323 (2007)

40. Annaby, M.H., Mansour, Z.S.: q-Fractional Calculus and Equations. Lecture Notes in Mathematics, vol. 2056. Springer, Heidelberg (2012)

41. Rajkovic, P.M., Marinkovic, S.D., Stankovic, M.S.: On q-analogues of Caputo derivative and Mittag-Leffler function. Fract. Calc. Appl. Anal. 10, 359-373 (2007)

\section{Submit your manuscript to a SpringerOpen ${ }^{0}$ journal and benefit from:}

- Convenient online submission

- Rigorous peer review

- Open access: articles freely available online

- High visibility within the field

- Retaining the copyright to your article

Submit your next manuscript at $>$ springeropen.com 